Vol. 74 (2006) [321-335]

\title{
TOPOLOGY OF FOUR-MANIFOLDS WTTH SPECIAL HOMOTOPY GROUPS
}

\section{Alberto Cavicchioli and Fulvia Spaggiari}

We study the homotopy type and the $s$-cobordism class of a closed connected topological 4-manifold with vanishing second homotopy group. Our results are related to problem 4.53 of Kirby in Geometric Topology, Studies in Advanced Math. 2 (1997), and give a partial answer to a question stated by Hillman in Bull. London Math. Soc. 27 (1995) 387-391.

\section{INTRODUCTION}

The first step to classify topological 4-manifolds is the determination of their homotopy type. The second step is to study the $s$-cobordism class to obtain at least theorems on the stable classification of 4-manifolds, that is, up to connected sums with copies of $\mathbb{S}^{2} \times \mathbb{S}^{2}$. The third step is the classification of the homeomorphism type. For this, one can use the surgery sequence [35] which was proved to be exact for a large class of fundamental groups. The proof for polycyclic groups and for elementary amenable groups follows from Freedman's early work $[11,12]$ (see also [13]). It was later generalised to subexponential groups $[14,15,25]$. A similar program has been realised (in part or entirely) for several families of topological closed 4-manifolds having special fundamental groups. We only mention some references in this direction. For topological closed 4-manifolds with finite fundamental groups we refer to $[1,3,4,16]$. Complete classifications are known when $\pi_{1}$ is cyclic. The homotopy type and the $s$-cobordism class of a closed topological 4-manifold with free fundamental group were classified in [6] and [8] (further developments can be found in $[19,24,27]$ ). Results on closed topological 4-manifolds with $\pi_{1}$-free second homotopy and on 4-manifolds having the fundamental group of an aspherical surface (respectively 4-manifold) were obtained in $[7,9,32]$. The stable classification of spin 4 -manifolds and splitting results (up to homotopy equivalence) for certain classes of 4 -manifolds were given in $[\mathbf{1 7}, \mathbf{3 3}]$.

The aim of this paper is to study the homotopy type and the $s$-cobordism class of closed topological 4-manifolds with vanishing second homotopy group.

One of the results we shall prove is the following

Received 14th March, 2006

Work performed under the auspices of the G.N.S.A.G.A. of the C.N.R. (National Research Council) of Italy and partially supported by the MIUR (Ministero Istruzione, Università e Ricerca) within the project "Proprietà Geometriche delle Varietà Reali e Complesse", and by a research grant of the University of Modena and Reggio Emilia.

Copyright Clearance Centre, Inc. Serial-fee code: 0004-9727/06 \$A2.00+0.00. 
THEOREM. Let $X^{4}$ be a closed connected orientable topological 4-manifold with $\pi_{1}(X)=A * F(r)$ and $\pi_{2}(X)=0$, where $F(r), r \geqslant 1$, denotes the free group of rank $r$, and $A$ has one-end. Let $Y$ be the closed 4-manifold obtained from $X$ by choosing a set of embedded loops representing the generators of the free part $F(r)$ of $\pi_{1}(X)$ and then doing surgeries on them. Then we have:

(1) The manifold $Y$ is aspherical;

(2) There exists a homotopy equivalence between $X$ and the connected sum $W$ of $Y$ with $r$ copies of $\mathbf{S}^{1} \times \mathbf{S}^{3}$;

(3) If $\mathrm{Wh}(A) \cong \mathrm{H}_{2}\left(A ; \mathbb{Z}_{2}\right) \cong 0$ and

$$
\sigma_{5}:[X \times I, \partial(X \times I), G / \text { TOP }] \rightarrow L_{5}\left(\pi_{1}(X)\right)
$$

is surjective, then $X$ and $W$ are $s$-cobordant (hence they become homeomorphic after taking connected sums with copies of $\mathbb{S}^{2} \times \mathbb{S}^{2}$ ).

The theorem is related to a question stated by Hillman in [19]: Let $X^{4}$ be a closed orientable topological 4-manifold with $\pi_{1}(X)$ torsion free and infinite, and $\pi_{2}(X)=0$. Is $X$ s-cobordant to a connected sum of aspherical 4-manifolds with copies of $\mathbb{S}^{1} \times \mathbb{S}^{3}$ ? Our result partially answers the question, and describes explicitly the aspherical summand of the connected sum. The techniques involved in our proofs are based on obstruction arguments, (co)homological algebra with local coefficients, and surgery theory. Examples of closed topological 4-manifolds satisfying the algebraic conditions of the theorem are explicitly constructed.

\section{FOUR-MANIFOLDS WITH VANISHING SECOND HOMOTOPY}

This section is devoted to studying the homotopy type and the $s$-cobordism class of a closed connected topological 4-manifold $M^{4}$ such that $\pi_{2}(M)=0$ (results on 4-manifolds with vanishing second homology can be found in [10]). Let $\Lambda=\mathbb{Z}\left[\pi_{1}(M)\right]$ denote the group ring of $\pi=\pi_{1}(M)$. The right action of $\pi$ on the chains of the universal cover $\widetilde{M}$ of $M$ induces a left $\Lambda$-module structure on $H^{*}(M ; \Lambda)$. The group ring $\Lambda$ has a canonical anti-involution defined by sending $g$ to $\bar{g}=w_{1}(g) g^{-1}$. (Here $w_{1}=w_{1}(M)$ denotes the first Stiefel-Whitney class considered as a homomorphism from $\pi_{1}(M)$ to $\mathbb{Z}_{2}$; it sends loops preserving orientation to +1 and those reversing orientation to -1 , where $\left.\mathbb{Z}_{2}=\{ \pm 1\}\right)$. This map induces a right $\Lambda$-module structure on $H^{*}(M ; \Lambda)$, denoted by $\bar{H}^{*}(M ; \Lambda)$.

Lemia 2.1. Let $M^{4}$ be a closed connected topological 4-manifold such that $\pi_{2}(M)=0$. Then we have

(1) $\bar{H}^{1}(\pi ; \Lambda) \cong \pi_{3}(M)$, where $\pi=\pi_{1}(M)$

(2) $\bar{H}^{2}(\pi ; \Lambda) \cong 0$

(3) $H_{2}\left(\pi ; \mathbb{Z}_{2}\right) \cong H_{2}\left(M ; \mathbb{Z}_{2}\right)$ 
Proof: (1) follows from Poincaré duality and the Hurewicz isomorphism. In fact we have

$$
\pi_{3}(M) \cong \pi_{3}(\widetilde{M}) \cong H_{3}(\widetilde{M}) \cong H_{3}(M ; \Lambda) \cong \bar{H}^{1}(M ; \Lambda) \cong \bar{H}^{1}(\pi ; \Lambda)
$$

(2) follows from the exact sequence in [18, Lemma 4, p. 16]:

$$
0 \longrightarrow \bar{H}^{2}(\pi ; \Lambda) \longrightarrow \pi_{2}(M) \longrightarrow \overline{\operatorname{Hom}}_{\Lambda}\left(\pi_{2}(M), \Lambda\right)
$$

(3) follows by noting that $K(\pi, 1)$ is homotopy equivalent to $M$ plus cells of dimension $\geqslant 4$.

For general references on the theory of groups see for example $[5,29]$. Concerning connections between group theory and the algebraic characterisation of four-manifolds see $[18,21]$. Here we briefly summarise some classical facts about ends of groups. Recall that a finitely generated group $\pi$ has $0,1,2$ or infinitely many ends. It has 0 ends if and only if it is finite, in which case $H^{0}(\pi ; \Lambda) \cong \mathbb{Z}$ and $H^{i}(\pi ; \Lambda) \cong 0$ for every $i \geqslant 1$. Otherwise, $H^{0}(\pi ; \Lambda) \cong 0$ and $H^{1}(\pi ; \Lambda)$ is a free Abelian group of rank $e(\pi)-1$, where $e(\pi)$ is the number of ends of $\pi$ (see [34]). The group $\pi$ has more than one end if and only if it is either a nontrivial generalised free product with amalgamation $\pi \cong A *_{C} B$ or an HNN extension $A *_{C} \phi$, where $C$ is a finite group, and $A \neq C \neq B$. In particular, it has two ends if and only if it is virtually $\mathbb{Z}$ if and only if it has a maximal finite normal subgroup $G$ such the quotient $\pi / G$ is either $\mathbb{Z}$ or $\mathbb{Z}_{2} * \mathbb{Z}_{2}$ (infinite dihedral). Any group with infinitely many ends has nonabelian free subgroups (hence it cannot be elementary amenable).

LemMa 2.2. Let $M^{4}$ be a closed connected topological 4-manifold such that $\pi_{2}(M)=0$. If $\pi=\pi_{1}(M)$ is finite, then $M$ is homeomorphic to either $\mathrm{S}^{4}, \mathbb{R} P^{4}$ or the unique non-smoothable homotopy $\mathbb{R} P^{4}$.

Proof: If $\pi$ is finite, then $\widetilde{M}$ is a closed 4-manifold. By Lemma 2.1 (1), we have $\pi_{3}(M) \cong H_{3}(\widetilde{M}) \cong 0$, so $\widetilde{M}$ is homotopy equivalent to $\mathbf{S}^{4}$. Then $\widetilde{M}$ is homeomorphic to $S^{4}$ by a celebrated theorem of Freedman (see for example [13, Corollary 7.1B, p. 102]). Therefore the only possibilities for $M$ are the finite quotients of $\mathrm{S}^{4}$. It is well-known that $\mathbb{Z}_{2}$ is the only non-trivial finite group that can act freely on $\mathbf{S}^{4}$ (see for example [21, p. 234]). Again by [13] $M$ must be homeomorphic to either $S^{4}, \mathbb{R} P^{4}$ or the unique non-smoothable homotopy $\mathbf{R} P^{4}$. Surgery theory establishes the existence of such a homotopy $\mathbb{R} P^{4}$, while Ruberman gave an explicit construction of it (see [30]). We recall that Freedman proved that every smooth fake $\mathbf{R} P^{4}$ is homeomorphic to the standard $\mathbb{R} P^{4}$. Finally, we remark that the non-smoothable homotopy $\mathbb{R} P^{4}$ cannot be expressed as $* \mathbf{R} P^{4}$ (see $[13$, Section 10.3], for this notation) since the latter is formally $\mathbf{R} P^{4}$ by $[13$, Section 10.4 , pp. 166-167]. 
LEMMA 2.3. Let $M^{4}$ be a closed connected topological 4-manifold such that $\pi_{2}(M)=0$. Then we have

(1) If $\pi=\pi_{1}(M)$ has one end, then $M$ is aspherical;

(2) If $\pi$ has two ends, then $M$ is finitely covered by $\mathbf{S}^{1} \times \mathbf{S}^{3}$. If further $\pi$ is torsion free, then $M$ is homeomorphic to either $\mathbf{S}^{1} \times \mathbf{S}^{3}$ or the twisted $\mathbf{S}^{3}$-bundle over $\mathbf{S}^{1}$.

Proof: (1) By Lemma $2.1(1)$, we have $H_{3}(\widetilde{M}) \cong \bar{H}^{1}(\pi ; \Lambda) \cong 0$. Furthermore, $H_{4}(\widetilde{M}) \cong 0$ since $\pi$ is infinite. Thus $\widetilde{M}$ is contractible, and $M \simeq K(\pi, 1)$.

(2) In this case we have $\pi_{i}(\widetilde{M}) \cong 0, i \leqslant 2, \pi_{3}(\widetilde{M}) \cong H_{3}(\widetilde{M}) \cong \bar{H}^{1}(\pi ; \Lambda) \cong \mathbb{Z}$, and $H_{4}(\widetilde{M}) \cong 0$. Then $\widetilde{M}$ is homotopy equivalent to $\mathrm{S}^{3}$. By $[18$, Theorem 10, p. 23 , and Theorem 1, p. 119], it follows that $M$ is finitely covered by $\mathbb{S}^{1} \times \mathbb{S}^{3}$. If $\pi$ is torsion free, then $M$ is homotopy equivalent to either $\mathbf{S}^{1} \times \mathbb{S}^{3}$ or the twisted $\mathbb{S}^{3}$-bundle over $\mathbb{S}^{1}$. Then $M$ is homeomorphic to one of these manifolds by surgery over $\mathbb{Z}$. Here we use the Freedman surgery theorem [13], and an extension of it for the nonorientable case (see [23, Theorem F, p. 709], and [36]).

The statement of the next theorem uses the first $k$-invariant of a manifold for which we refer for example to [18, p. 14], and [37, p. 421].

THEOREM 2.4. Let $M$ and $N$ be closed connected topological 4-manifolds with $\pi_{2}(M)=\pi_{2}(N) \doteq 0$. Then $M$ and $N$ are homotopy equivalent if and only if there is an isomorphism $\theta: \pi_{1}(M) \rightarrow \pi_{1}(N)$ such that $w_{1}(N) \circ \theta=w_{1}(M)$ and $\theta^{*} k_{1}(N)=k_{1}(M)$, where $w_{1}$ and $k_{1}$ are the first Stiefel-Whitney class and the first $k$-invariant of the considered manifolds.

Proof: Let $P_{3}(M)$ be the third stage of a Postnikov tower for $M$, and

$$
c_{M}: M \rightarrow K\left(\pi_{1}(M), 1\right)
$$

the classifying map for the universal covering of $M$. Let $f_{M}: M \rightarrow P_{3}(M)$ be a 4-equivalence, that is, $f_{M}$ induces isomorphisms on $\pi_{i}$ for every $i \leqslant 3$. Let $c_{M}=c_{P_{3}(M)} \circ f_{M}$ be a factorisation of $c_{M}$ through $P_{3}(M)$. A map

$$
g: X \rightarrow K\left(\pi_{1}(M), 1\right)
$$

lifts to a map from $X$ to $P_{3}(M)$ if and only if $g^{*} k_{1}(M)=0$, where $k_{1}(M)$ $\in H^{4}\left(\pi_{1}(M) ; \pi_{3}(F)\right)$ is the first $k$-invariant of $M$. Since $\pi_{2}(M)=0$, the fibre $F$ of the fibration

$$
F \longrightarrow P_{3}(M) \longrightarrow K\left(\pi_{1}(M), 1\right)
$$

is homotopy equivalent to $K\left(\pi_{3}(M), 3\right)$, hence $\pi_{3}(F) \cong \pi_{3}(M)$. In our case, any isomorphism $\left[\pi_{1}, \pi_{2}, k\right] \cong\left[\pi_{1}^{\prime}, \pi_{2}^{\prime}, k^{\prime}\right]$ of algebraic 2-types can be realised by a homotopy 
equivalence between $P_{3}(M)$ and $P_{3}\left(M^{\prime}\right)$. For the concept of algebraic 2-type see for example [16] and [18, p. 14]. The hypothesis imply that there is a map $h_{3}: P_{3}(M)$ $\rightarrow P_{3}(N)$ which induces isomorphisms on $\pi_{i}$ for every $i \leqslant 3$. Since $P_{3}(N)$ is obtained from $N$ by attaching cells of dimension $\geqslant 5$, the cellular approximation theorem gives a map $h: M \rightarrow N$ such that the diagram

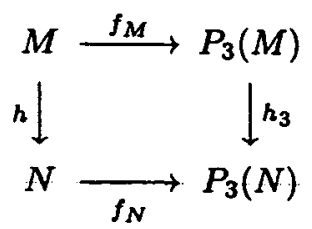

commutes. Then $h$ induces isomorphisms on $\pi_{i}$ for every $i \leqslant 3$. Hence $\tilde{f}: \widetilde{M} \rightarrow \tilde{N}$ is a homotopy equivalence (by using the theorems of Hurewicz and Whitehead). This implies that $h$ is a homotopy equivalence.

Let $M^{4}$ be a closed connected orientable topological 4-manifold. Let $\mathrm{HE}_{\text {id }}(M)$ denote the set of homotopy classes of (simple) self-homotopy equivalences of $M$ which induce the identities on $\pi=\pi_{1}(M)$ and on $H_{*}=H_{*}(M)$. Let $\mathcal{N}_{4}^{\text {TOP }}(M)$ be the set of normal invariants of $M$, and $L_{4}(\pi)$ the 4-th Wall group (as a general reference on surgery theory of compact manifolds see [35]).

The following result was proved in [8]

THEOREM 2.5. With the above notations, if $H_{2}\left(\pi ; \mathbb{Z}_{2}\right) \cong 0$, then the sequence

$$
\mathrm{HE}_{\text {id }}(M) \longrightarrow \mathcal{N}_{4}^{\mathrm{TOP}}(M) \stackrel{\sigma_{4}^{\mathrm{TOP}}}{\longrightarrow} L_{4}(\pi)
$$

is exact, where $\sigma_{4}^{\text {TOP }}$ is the surgery obstruction map in the topological category.

We apply Theorem 2.5 in our case, and we get

THEOREM 2.6. Suppose that $M$ and $N$ are closed orientable homotopy equivalent 4-manifolds with vanishing second homotopy groups. If $\mathrm{Wh}(\pi)=H_{2}\left(\pi ; \mathbb{Z}_{2}\right) \cong 0$ and $\sigma_{5}:[N \times I, \partial(N \times I), G / \mathrm{TOP}] \rightarrow L_{5}(\pi)$ is surjective, where $\pi=\pi_{1}(M)=\pi_{1}(N)$, then the manifolds are $s$-cobordant (and hence they become homeomorphic under a stabilisation with copies of $\mathbf{S}^{2} \times \mathbf{S}^{2}$ ). If further, $\pi$ is good, that is, a group for which the embedding theorem is known (see [11] and [12, p. 99]), then the manifolds are homeomorphic.

Proof: If $\mathrm{Wh}(\pi)=0$, any homotopy equivalence is simple, and $h$-cobordant is equivalent to $s$-cobordant. If $H_{2}\left(\pi ; \mathbb{Z}_{2}\right) \cong 0$, then the sequence in Theorem 2.5 is exact. By the Sullivan theorem, there is a bijection between $\mathcal{N}_{4}^{\text {TOP }}(M)$ and the group $[M, G /$ TOP $]$ of the homotopy classes of maps from $M$ to $G /$ TOP. Since 
$\pi_{2}(G /$ TOP $) \cong \mathbb{Z}_{2}, \pi_{3}(G /$ TOP $) \cong \pi_{5}(G /$ TOP $) \cong 0$ and $\pi_{4}(G /$ TOP $) \cong \mathbb{Z}$ with vanishing $k$-invariant in $H^{5}\left(K\left(\mathbb{Z}_{2}, 2\right)\right)$, the Postnikov resolution of $G /$ TOP gives a map $G /$ TOP $\rightarrow K\left(\mathbb{Z}_{2}, 2\right) \times K(\mathbb{Z}, 4)$ which is a 5 -equivalence, that is, we can assume that the 5 -skeleton of $G /$ TOP is the same as that of $K\left(\mathbb{Z}_{2}, 2\right) \times K(\mathbb{Z}, 4)$. (For the Sullivan theorem and the calculations of homotopy groups see for example [28, Chapter 5], and [35, p. 113]). Thus we have isomorphisms

$$
\begin{aligned}
\mathcal{N}_{4}^{T O P}(M) & \cong[M, G / \mathrm{TOP}] \cong\left[M, K\left(\mathbb{Z}_{2}, 2\right) \times K(\mathbb{Z}, 4)\right] \cong H^{2}\left(M ; \mathbb{Z}_{2}\right) \oplus H^{4}(M) \\
& \cong H_{2}\left(M ; \mathbb{Z}_{2}\right) \oplus \mathbb{Z} \cong H_{2}\left(\pi ; \mathbb{Z}_{2}\right) \oplus \mathbb{Z} \cong \mathbb{Z}
\end{aligned}
$$

by Lemma 2.1 (3) and the hypothesis $H_{2}\left(\pi ; \mathbb{Z}_{2}\right) \cong 0$. Then the signature difference gives a bijection from $\mathcal{N}_{4}^{\text {TOP }}(M) \cong \mathbb{Z}$ to $L_{4}(1) \cong \mathbb{Z} \subset L_{4}(\pi)$. Let $h: M \rightarrow N$ be a simple homotopy equivalence: by the above and using Theorem 2.5 there is a normal cobordism $H: P \rightarrow N \times I, I=[0,1]$, such that $\left.H\right|_{\partial_{-} P}=h$ and $\left.H\right|_{\partial_{+} P}=\operatorname{id}_{N}$. But $\sigma_{5}:[N \times I, \partial(N \times I), G /$ TOP $] \rightarrow L_{5}(\pi)$ is surjective, so there is another normal cobordism $H^{\prime}: P^{\prime} \rightarrow N \times I$ from id ${ }_{N}$ to itself with surgery obstruction $\sigma_{5}^{\text {TOP }}\left(P^{\prime}, H^{\prime}\right)$ $=-\sigma_{5}^{\text {TOP }}(P, H)$ in $L_{5}(\pi)$, by the argument of [13, Proposition 11.6B]. The union of these two normal cobordisms along $\partial_{+} P=\partial_{-} P^{\prime}$ is a normal cobordism from $h$ to $\mathrm{id}_{N}$ with vanishing surgery obstruction. So we may obtain an $s$-cobordism by 5 dimensional surgery theory ( $\mathrm{rel} \partial$ ). Finally, if $\pi$ is good, then the result follows from Theorem 7.1A of $[13$, p. 101] .

To complete the section we describe many examples of closed connected topological 4-manifolds with vanishing second homotopy.

1. SURGERY ON TWO-KNOTS Let $M=M(K)$ be the closed 4-manifold obtained by surgery on a 2 -knot $K$ in $\mathrm{S}^{4}$. Then $M$ is aspherical if and only if the knot group $\pi=\pi(K)$ is a $\mathrm{PD}_{4}^{+}$-group and the image of the orientation class of $M$ in $H_{4}(\pi ; \mathbb{Z})$ is non zero (use $[18$, Theorem 5, p. 16]). The argument has been extended to elementary amenable normal subgroups with restricted torsion (see Chapter $\mathrm{X}$ of [18]). In particular, if $\pi=\pi(K)$ is elementary amenable, then $M=M(K)$ is aspherical if and only if $\pi$ has one end and $H^{2}(\pi ; \Lambda) \cong 0$.

2. Products Let $M^{3}$ be a closed irreducible sufficiently large 3-manifold. Then we have $M^{3} \simeq K(\pi, 1)$ and $\mathrm{Wh}(\pi)=0$, where $\pi=\pi_{1}\left(M^{3}\right)$. Examples are given by hyperbolic 3-manifolds and aspherical Seifert fibred spaces. Then the closed 4-manifold $M^{3} \times S^{1}$ is aspherical with infinite torsion free fundamental group. Other examples of aspherical 4-manifolds are given by the topological products of two aspherical closed surfaces. It is known that many homology 3-spheres are $K(\pi, 1)$ ' $\mathrm{s}$, as for example the Brieskorn manifolds $\Sigma=\Sigma(p, q, r)$ with $p, q, r$ pairwise coprime and infinite fundamental group. Then the products $\Sigma \times \mathbb{S}^{1}$ are closed aspherical 4-manifolds. In this 
case, we have $H_{2}\left(\Sigma \times \mathbb{S}^{1} ; \mathbb{Z}\right) \cong H_{2}\left(\Sigma \times \mathbb{S}^{1} ; \mathbb{Z}_{2}\right) \cong 0$. For a topological product of a lens space $L=L(p, q)$ (including $\mathbb{S}^{3}$ and $\mathbb{S}^{2} \times \mathbb{S}^{1}$ ) with $\mathbb{S}^{1}$, we have $\pi_{1} \cong \mathbb{Z}_{p} \oplus \mathbb{Z}$, $\pi_{2} \cong 0, \pi_{3} \cong \mathbb{Z}, H_{2}\left(L \times \mathbb{S}^{1} ; \mathbb{Z}\right) \cong \mathbb{Z}_{p}$ and $H_{2}\left(L \times \mathbb{S}^{1} ; \mathbb{Z}_{2}\right) \cong \mathbb{Z}_{(p, 2)}^{2}$ (which is trivial for $p$ odd). Ruberman constructed in [31] a closed topological 4 -manifold which is simple homotopy equivalent to $L(3,1) \times \mathbf{S}^{1}$ but which contains no quotient of $\mathbf{S}^{3}$ (by a finite group acting linearly) representing a nontrivial element of $\pi_{3} \cong \mathbb{Z}$. Finally, let $H W$ denote the Hantzde-Wendt 3-manifold, which is orientable and flat with fundamental group presented by generators $x$ and $y$ and relations $x y^{2} x^{-1}=y^{-2}$ and $y x^{2} y^{-1}=x^{-2}$. Then $H W \times \mathbb{S}^{1}$ is orientable and aspherical with $H_{2}\left(H W \times \mathbb{S}^{2} ; \mathbb{Z}\right) \cong \mathbb{Z}_{4}^{2}$ and $H_{2}\left(H W \times \mathbb{S}^{1} ; \mathbb{Z}_{2}\right) \cong \mathbb{Z}_{2}^{4}$.

3. Homology 4- SPHERES In [22, Problem 4.17, p. 252], one asks for examples of homology 4-spheres which are $K(\pi, 1)$ 's. Examples of rational homology 4-spheres which are aspherical were constructed by Luo in [26].

4. BundLes $\mathbb{S}^{1}$-bundles over closed irreducible 3 -manifolds are examples of closed 4-manifolds with $\pi_{2} \cong 0$. Surface-bundles over aspherical surfaces with aspherical fibres are examples of closed 4-manifolds with $\pi_{2} \cong 0$. A study of the homotopy type and the $s$-cobordism class of a closed 4-manifold with surface fundamental group can be found in [9]. Aspherical complex surfaces which are fibre bundles have been characterised in [20] by using their Euler characteristics and fundamental groups.

5. FOUR-MANIFOLDS COVERED BY $\mathbb{S}^{3} \times \mathbb{R}$ These are examples of manifolds with $\pi_{2} \cong 0$. This class was deeply studied by Hillman, and a lot of interesting results concerning their topological and geometrical structures can be found in [18, Chapter VIII] and [21, Chapter 11]. We only mention an interesting example. For any $n>1$ odd and $(s, n)=1$, let us consider the group

$$
\pi=\mathbb{Z}_{n} \times_{s} \mathbb{Z}=\left\langle x, t: \quad x^{n}=1, \quad t x t^{-1}=x^{s}\right\rangle
$$

whose Abelianisation is $\mathbb{Z}_{(n, s-1)} \oplus \mathbb{Z}$. All such groups are realised by geometric 4-manifolds $M$ which fibre over $\mathbb{S}^{1}$ with fibre a lens space. In particular, we have $\chi(M)=0, \widetilde{M} \cong \mathbb{S}^{3} \times \mathbb{R}, H_{2}(M ; \mathbb{Z}) \cong \mathbb{Z}_{(n, 8-1)}$ and $H_{2}\left(M ; \mathbb{Z}_{2}\right) \cong 0$.

\section{HOMOTOPY TYPE, COBORDISM AND STABLE CLASSIFICATION}

To complete the study of closed 4-manifolds $M$ with $\pi_{2}(M) \cong 0$ there remains the case when $\pi=\pi_{1}(M)$ has infinitely many ends. Then $\pi$ is isomorphic to either $A *_{C} B$ or $A *_{C} \phi$, where $C$ is finite, and either $[A: C]>2$, or $[B: C]>2$, or $[A: \phi(C)]>2$. If $\pi$ is torsion free, then we must have $C=1$, so $\pi \cong A * B$ or $A * \mathbb{Z}$. This implies that $\pi \cong A_{1} * \cdots * A_{p} * F(r)$, where each $A_{i}$ has one end. Here we shall consider the case $\pi \cong A * F(r), r \geqslant 1$, where $A$ has one end, and prove the theorem stated in Section 1 . 
From now on, let $X$ denote a closed connected orientable topological 4-manifold such that $\pi=A * F(r), r \geqslant 1$, and $\pi_{2}=0$, where $A$ has one end. Let $Y$ be the closed 4-manifold obtained from $X$ by choosing a set of embedded loops representing the generators of the free part $F(r)$ of $\pi$ and then doing surgeries on them.

LEMMA 3.1. The manifold $Y$ is aspherical, that is, $Y \simeq K(A, 1)$.

Proof: Let $\gamma_{1}, \ldots, \gamma_{r}: S^{1} \times D^{3} \rightarrow X$ be embeddings representing the generators of the free part of $\pi_{1}(X)$, and denote by $\Gamma$ the normal subgroup generated by $\left[\gamma_{1}\right], \ldots$, $\left[\gamma_{r}\right] \in \pi_{1}(X)$. Put $A=\pi_{1}(X) / \Gamma$, and let $\widehat{X} \rightarrow X$ be the covering with $\pi_{1}(\widehat{X})=\Gamma$. Doing surgeries on $\gamma_{1}, \ldots, \gamma_{r}$ we denote

$$
X_{0}=X \backslash \bigcup_{i=1}^{r} \gamma_{i}\left(\mathbf{S}^{1} \times D^{3}\right) \quad \text { and } \quad Y=X_{0} \cup \bigcup_{i=1}^{r}\left(D^{2} \times \mathbb{S}^{2}\right) .
$$

This is equivalent to doing $A$-equivariant surgeries on $\widehat{X}$. Let

$$
\hat{X}_{0}=\widehat{X} \backslash \bigcup_{\substack{i=1 \\ a \in A}}^{r} \gamma_{i}^{a}\left(\mathbb{S}^{1} \times D^{3}\right) \quad \text { and } \quad \hat{Y}=\hat{X}_{0} \cup \bigcup_{\substack{i=1 \\ a \in A}}^{r}\left(D^{2} \times \mathbb{S}^{2}\right) .
$$

Then $\pi_{1}(\widehat{Y}) \cong 0$, that is, $\widehat{Y} \rightarrow Y$ is the universal covering space. Note that

$$
\pi_{1}\left(\widehat{X}_{0}\right) \longrightarrow \pi_{1}(\widehat{X})=\Gamma
$$

and

$$
0 \rightarrow H_{2}\left(\widehat{X}_{0} ; \mathbb{Z}[\Gamma]\right) \rightarrow H_{2}(\widehat{X} ; \mathbb{Z}[\Gamma]) \rightarrow H_{2}\left(\widehat{X}, \widehat{X}_{0} ; \mathbb{Z}[\Gamma]\right) \rightarrow 0
$$

is exact (see Sublemma 2 below). By (1) the sequence in (2) can also be written

$$
0 \longrightarrow \pi_{2}\left(\widehat{X}_{0}\right) \longrightarrow \pi_{2}(\hat{X}) \longrightarrow \pi_{2}\left(\widehat{X}, \widehat{X}_{0}\right) \longrightarrow 0 .
$$

Since $\pi_{2}(\hat{X}) \cong \pi_{2}(X)=0$, we get $\pi_{2}\left(\widehat{X}_{0}\right)=0$. So we obtain the exact sequence

$$
0 \longrightarrow \pi_{2}(\hat{Y}) \longrightarrow \pi_{2}\left(\widehat{Y}, \hat{X}_{0}\right) \longrightarrow \pi_{1}\left(\widehat{X}_{0}\right) \cong \Gamma \longrightarrow 0 .
$$

SUbLemma 1. If $\pi_{1}(X)=A * F(r)$ with $\left[\gamma_{1}\right], \ldots,\left[\gamma_{r}\right] \in F(r)$ free generators, then the homomorphism

$$
\pi_{2}\left(\widehat{Y}, \widehat{X}_{0}\right) \rightarrow \pi_{1}\left(\widehat{X}_{0}\right) \cong \pi_{1}(\widehat{X})=\Gamma
$$


is injective.

Proof: The space $\widehat{Y}$ is obtained by adjoining $D^{2} \times \mathbb{S}^{2}$ 's to $\widehat{X}_{0}$ and attaching them by $\gamma_{i}^{a}, a \in A$. Then $\pi_{2}\left(\widehat{Y}, \widehat{X}_{0}\right)$ is generated by elements $\beta_{i}^{a}$ corresponding to $\gamma_{i}^{a}$, for $i=1, \ldots, r$ and $a \in A$. These elements are mapped to $\gamma_{i}^{a} \in \pi_{1}(\widehat{X})=\Gamma \subset A * F(r)$. This implies the injectivity of the map $\pi_{2}\left(\widehat{Y}, \widehat{X}_{0}\right) \rightarrow \pi_{1}\left(\widehat{X}_{0}\right)$.

Sublemma 1 and exact sequence (4) imply that

$$
\pi_{2}(Y) \cong \pi_{2}(\widehat{Y}) \cong 0
$$

The statement of Lemma 3.1 follows then from Lemma 2.3.

Sublemma 2. If $\pi_{1}(X)=A * F(r)$ and $H^{1}(B A ; \mathbb{Z}[A])=0$, then sequence (2)

$$
0 \longrightarrow H_{2}\left(\widehat{X}_{0} ; \mathbb{Z}[\Gamma]\right) \longrightarrow H_{2}(\widehat{X} ; \mathbb{Z}[\Gamma]) \longrightarrow H_{2}\left(\widehat{X}, \widehat{X}_{0} ; \mathbb{Z}[\Gamma]\right) \longrightarrow 0
$$

is exact.

ProOF: Since (2) is equivalent to (3), and $\pi_{q}(\widehat{X}) \cong \pi_{q}(\tilde{X}), \pi_{q}\left(\widehat{X}, \widehat{X}_{0}\right)$ $\cong \pi_{q}\left(\tilde{X}, \tilde{X}_{0}\right)$ for the universal covering $\widetilde{X} \rightarrow X$ and $q \geqslant 2$, it suffices to prove that the sequence

$$
H_{3}(X ; \Lambda) \longrightarrow H_{3}\left(X, X_{0} ; \Lambda\right) \longrightarrow 0
$$

is exact, where $\Lambda=\mathbb{Z}\left[\pi_{1}(X)\right]$. This follows from the diagram

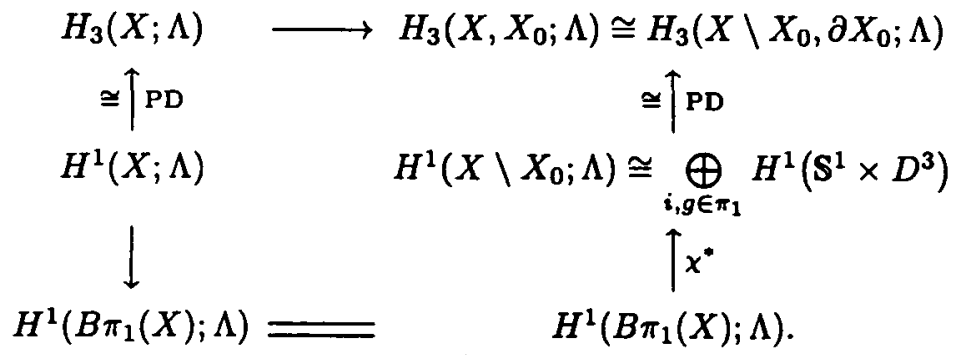

Of course, we have isomorphisms

$$
H^{1}\left(B \pi_{1}(X) ; \Lambda\right) \cong H^{1}\left(B A \vee \bigvee_{i=1}^{r} \mathbf{S}^{1} ; \Lambda\right) \cong H^{1}(B A ; \Lambda) \oplus H^{1}\left(\bigvee_{i=1}^{r} \mathrm{~S}^{1} ; \Lambda\right)
$$

But $H^{1}(B A ; \mathbb{Z}[A]) \cong 0$ implies $H^{1}(B A ; \Lambda) \cong 0$. Hence the map

$$
\chi: X \backslash X_{0} \rightarrow B \pi_{1}(X)
$$

induces an isomorphism $\chi^{*}: H^{1}\left(B \pi_{1}(X) ; \Lambda\right) \rightarrow H^{1}\left(X \backslash X_{0} ; \Lambda\right)$. In other words, the map $H_{3}(X ; \Lambda) \rightarrow H_{3}\left(X, X_{0} ; \Lambda\right)$ is an isomorphism. This proves the sublemma. 
SUPPLEMENT. Obviously $H_{2}\left(\widehat{X}, \widehat{X}_{0} ; \mathbb{Z}[\Gamma]\right) \cong 0$, so we have under the above hypotheses

$$
\pi_{2}\left(\widehat{X}_{0}\right) \longrightarrow \pi_{2}(\widehat{X}) \text {. }
$$

LEMMA 3.2. There is a degree one map $f: X \rightarrow Y$.

Proof: Let $\gamma_{i}: \mathbf{S}^{1} \times D^{3} \rightarrow X, i=1, \ldots, r$, be the embeddings on which surgeries are done to obtain $Y$. Then

$$
\Omega^{5}=(X \times I) \bigcup_{U \gamma_{i}}\left(\bigcup_{i=1}^{r} D^{2} \times D^{3}\right)
$$

is the trace cobordism with $\partial_{0} \Omega=X$ and $\partial_{1} \Omega=Y$. Seen from the opposite side, we have

$$
\Omega=(Y \times I) \bigcup_{\cup \widehat{\gamma}_{i}}\left(\bigcup_{i=1}^{r} D^{3} \times D^{2}\right),
$$

where $\left.\widehat{\gamma}_{i}\right|_{\mathbf{S}^{2} \times 0}$ is the transverse sphere of $\gamma_{i}$. Since $Y \simeq K(A, 1)$, the identity map of $Y$ extends to $\Omega$, that is, $Y$ is a retract of $\Omega$. If $\rho: \Omega \rightarrow Y$ is a retraction, then the composite map

$$
f:=\rho \circ j: X \stackrel{j}{\longrightarrow} \Omega \stackrel{\rho}{\longrightarrow} Y
$$

is a degree one map.

THEOREM 3.3. The manifold $X$ is homotopy equivalent to $W=Y \# r\left(\mathbb{S}^{1} \times \mathbb{S}^{3}\right)$.

Proof: Let $e_{1}, \ldots, e_{r} \in H_{1}(X ; \mathbb{Z})$ be the elements given by $\gamma_{1} \mid \mathbf{s}^{1} \times 0, \ldots$, $\gamma_{r} \mid \mathbf{s}^{1} \times 0$, respectively. Denote by

$$
u_{1}, \ldots, u_{r} \in H^{1}(X ; \mathbb{Z}) \cong \operatorname{Hom}_{\mathbf{Z}}\left(H_{1}(X ; \mathbb{Z}), \mathbb{Z}\right)
$$

the "dual elements" of $e_{1}, \ldots, e_{r}$, that is, complete $e_{1}, \ldots, e_{r} \in H_{1}(X ; \mathbb{Z}) /$ TOR (here TOR $=0$ ) to a basis, and select from the dual basis the elements $u_{1}, \ldots, u_{r}$ dual to $e_{1}, \ldots, e_{r}$. Here $u_{i}$ are represented by maps $u_{i}: X \rightarrow \mathbb{S}^{1}=K(\mathbb{Z}, 1)$. Let

$$
v_{1}, \ldots, v_{r} \in H^{3}(X ; \mathbb{Z})
$$

be the Poincaré duals of the elements $e_{1}, \ldots, e_{r}$. Here $v_{i}$ are represented by maps $v_{i}: X \rightarrow K(\mathbb{Z}, 3)$. Then the map

$$
\bar{g}:=\prod_{i=1}^{r}\left(u_{i} \times v_{i}\right): X \rightarrow \prod_{i=1}^{r} \mathrm{~s}^{1} \times K(\mathbb{Z}, 3)
$$

factors over

$$
g: X \rightarrow \prod_{i=1}^{r}\left(\mathbb{S}^{1} \times \mathbb{S}^{3}\right) .
$$

Now we need the following 
Sublemma. The map

$$
f \times g: X \rightarrow Y \times \prod_{i=1}^{r}\left(\mathbf{S}^{1} \times \mathbf{S}^{3}\right)
$$

induces an isomorphism

$$
\pi_{3}(X) \otimes_{\Lambda} \mathbb{Z} \rightarrow \bigoplus_{i=1}^{r} \pi_{3}\left(\mathbf{S}^{1} \times \mathbf{S}^{3}\right) \cong \mathbb{Z}^{r}
$$

(note that $\pi_{3}(Y)=0$ ).

Proof: Consider the spectral sequence

$$
E_{p q}^{2}=\operatorname{Tor}_{p}^{\Lambda}\left(H_{q}(X ; \Lambda), \mathbb{Z}\right) \Rightarrow H_{p+q}(X) .
$$

Since $\pi_{2}(X)=0$, we have $E_{p q}^{2}=0$ for $q=1,2$ and arbitrary $p$. Further,

$$
E_{30}^{\infty} \cong H_{3}\left(B \pi_{1}(X) ; \mathbb{Z}\right)
$$

and

$$
d_{40}^{2}: E_{40}^{2} \rightarrow E_{03}^{2} \cong H_{3}(X ; \Lambda) \otimes_{\Lambda} \mathbb{Z}
$$

Hence we get

$$
H_{3}(X ; \mathbb{Z}) \cong E_{30}^{\infty} \oplus E_{03}^{\infty} \cong H_{3}\left(B \pi_{1}(X) ; \mathbb{Z}\right) \oplus E_{03}^{2} / \operatorname{Im} d_{40} .
$$

But $d_{40}=0$ because

$$
H_{4}(X ; \mathbb{Z}) \rightarrow E_{40}^{\infty}=\operatorname{Ker} d_{40} \subset H_{4}\left(B \pi_{1}(X) ; \mathbb{Z}\right)
$$

is the classifying map. Since

$$
B \pi_{1}(X)=Y \vee\left(\bigvee_{i=1}^{r} \mathrm{~s}^{1}\right)
$$

and the map

$$
H_{4}(X) \rightarrow H_{4}\left(B \pi_{1}(X)\right) \cong H_{4}(Y)
$$

is just $f_{*}$, it follows that $\operatorname{Ker} d_{40} \neq 0$. But $H_{4}\left(B \pi_{1}(X)\right) \cong \mathbb{Z}$, and $d_{40}=0$, hence we have the diagram

$$
\begin{aligned}
E_{03}^{2} \cong H_{3}(X ; \Lambda) \otimes_{\Lambda} \mathbb{Z} \cong \pi_{3}(X) \otimes_{\Lambda} \mathbb{Z} & \longrightarrow \prod_{i=1}^{r} \pi_{3}\left(\mathbf{S}^{1} \times \mathbf{S}^{3}\right) \\
\downarrow & \downarrow \cong \\
H_{3}(X ; \mathbb{Z}) \cong H_{3}\left(B \pi_{1}(X) ; \mathbb{Z}\right) \oplus \prod_{i=1}^{r} H_{3}\left(\mathbf{S}^{1} \times \mathbf{S}^{3}\right) & \longrightarrow \prod_{i=1}^{r} H_{3}\left(\mathbf{S}^{1} \times \mathbf{S}^{3}\right)
\end{aligned}
$$


where $H_{3}\left(B \pi_{1}(X) ; \mathbb{Z}\right) \cong H_{3}(Y)$. This implies the statement of the sublemma.

Now we consider $W=Y \# r\left(\mathbf{S}^{1} \times \mathbf{S}^{3}\right)$. Obviously, $\pi_{1}(X)=\pi_{1}(W)$, and $B \pi_{1}(X)$ is obtained from $W$ as follows. First build

$$
W^{*}=W \cup\left(D^{4} \cup D_{1}^{4} \cup \cdots \cup D_{r-1}^{4}\right) \simeq Y \vee\left(\bigvee_{i=1}^{r} \mathbf{s}^{1} \times \mathbf{S}^{3}\right)
$$

Then adjoin a 4-disc to each component $\mathbf{S}^{1} \times \mathbf{S}^{3}$, that is, $\mathbf{S}^{1} \times \mathbb{S}^{3} \cup\{1\} \times D^{4} \simeq_{\text {h.e. }}$. $\mathbf{S}^{1} \vee \mathbf{S}^{4}$. Finally, fill a 5-disc $D^{5}$ to each component $\mathbf{S}^{1} \vee \mathbf{S}^{4}$. In particular, we have $\left[B \pi_{1}(X)\right]^{(3)}=W^{(3)}$.

Let $h: X \rightarrow B \pi_{1}(X)$ be the classifying map. By the cellular approximation theorem we get

$$
h^{(3)}: X^{(3)} \rightarrow W^{(3)}
$$

Consider the following maps

$$
X^{(3)} \stackrel{h^{(3)}}{\longrightarrow} W \longrightarrow Y \vee\left(\bigvee_{i=1}^{r} \mathbf{S}^{1} \times \mathbf{S}^{3}\right) \longrightarrow Y \times \prod_{i=1}^{r}\left(\mathbb{S}^{1} \times \mathbb{S}^{3}\right)
$$

and

$$
X^{(3)} \subset X \stackrel{f \times g}{\longrightarrow} Y \times \prod_{i=1}^{r}\left(\mathbf{S}^{1} \times \mathbb{S}^{3}\right)
$$

Let

$$
\Delta: H_{3}\left(\tilde{X}^{(3)}, \widetilde{X}^{(2)}\right) \rightarrow \pi_{3}\left(Y \times \prod_{i=1}^{r}\left(\mathbf{S}^{1} \times \mathbf{S}^{3}\right)\right)
$$

be the difference cochain of the above maps (see [2, Theorem 4.2.14]). Since

$$
\pi_{3}(W) \rightarrow \pi_{3}\left(Y \times \prod_{i=1}^{r}\left(\mathbf{S}^{1} \times \mathbf{S}^{3}\right)\right)
$$

is surjective, the map $\Delta$ lifts equivariantly to

$$
\widetilde{\Delta}: H_{3}\left(\widetilde{X}^{(3)}, \tilde{X}^{(2)}\right) \rightarrow \pi_{3}(W) .
$$

Then $h^{(3)}$ can be changed by $\widetilde{\Delta}$ to give a map

$$
\left(h^{\prime}\right)^{(3)}: X^{(3)} \rightarrow W
$$

such that the composition

$$
X^{(3)} \longrightarrow W \longrightarrow Y \vee\left(\bigvee_{i=1}^{r} \mathbf{S}^{1} \times \mathbb{S}^{3}\right) \subset Y \times \prod_{i=1}^{r}\left(\mathbb{S}^{1} \times \mathbb{S}^{3}\right)
$$


is homotopic to

$$
X^{(3)} \subset X \stackrel{f \times g}{\longrightarrow} Y \times \prod_{i=1}^{r}\left(\mathbf{S}^{1} \times \mathbb{S}^{3}\right) .
$$

The obstruction for extending $\left(h^{\prime}\right)^{(3)}$ to $X$ belongs to

$$
H^{4}\left(X ; \pi_{3}(W)\right) \cong H_{0}\left(X ; \pi_{3}(W)\right) \cong \pi_{3}(W) \otimes_{\Lambda} \mathbb{Z} \cong \bigoplus_{i=1}^{r} \pi_{3}\left(\mathbf{S}^{1} \times \mathbf{S}^{3}\right)
$$

by using the sublemma. By the choice of $\left(h^{\prime}\right)^{(3)} \simeq(f \times g)^{(3)}$, the obstruction is zero since $g$ defines an extension. Thus $X \simeq W$, as claimed.

THEOREM 3.4. If

$$
W h(A) \cong H_{2}\left(A ; \mathbb{Z}_{2}\right) \cong 0
$$

and

$$
\sigma_{5}:[X \times I, \partial(X \times I), G / \mathrm{TOP}] \rightarrow L_{5}(\pi)
$$

is surjective, then $X$ and $W=Y \# r\left(\mathbf{S}^{1} \times \mathbb{S}^{3}\right)$ are $s$-cobordant, hence they are stably homeomorphic.

Proof: We have

$$
H_{2}\left(\pi ; \mathbb{Z}_{2}\right) \cong H_{2}\left(Y \vee\left(\bigvee_{i=1}^{r} \mathbb{S}^{1}\right) ; \mathbb{Z}_{2}\right) \cong H_{2}\left(Y ; \mathbb{Z}_{2}\right) \cong H_{2}\left(A ; \mathbb{Z}_{2}\right) \cong 0
$$

and

$$
W h(\pi)=W h(A * F(r))=W h(A) \oplus W h(F(r))=W h(A) \oplus W h(\mathbb{Z})^{r} \cong W h(A)
$$

as $W h(\mathbb{Z})=0$. Now we can apply Theorem 2.6.

Examples of groups with vanishing Whitehead torsion are given by torsion free poly-(finite or cyclic) groups (see $[13$, p. 101] or $[18$, p. 68]). A torsion free poly$\mathbb{Z}$ group of Hirsch length 4 is the fundamental group of an infrasolvmanifold $M$ of dimension 4 (see [18, Chapter VI], and [21, Chapter 7]). Such a manifold is aspherical with $\chi(M)=0$ and one-ended $\pi_{1}(M)$. Aspherical 4-manifolds with poly-(finite or cyclic) fundamental groups were also considered in [13, Section 11.5, p. 205].

\section{REFERENCES}

[1] S. Bauer, 'The homotopy type of a 4-manifold with finite fundamental group', in Lect. Notes in Math. 1361 (Springer-Verlag, Berlin, Heidelberg, New York, 1988), pp. 1-6.

[2] H.J. Baues, Obstruction theory, Lect. Notes in Math. 628 (Springer-Verlag, Berlin, Heidelberg, New York, 1977). 
[3] H.J. Baues, Combinatorial Homotopy and 4-Dimensional Complexes (Walter de Gruyter, Berlin, New York, 1991).

[4] H.J. Baues, Homotopy type and homology, Oxford Science Publ. (Clarendon Press, Oxford, 1996).

[5] R. Bieri, Homological dimension of discrete groups (Queen Mary College Mathematics Notes, London, 1976).

[6] A. Cavicchioli and F. Hegenbarth, 'On 4-manifolds with free fundamental group', Forum Math. 6 (1994), 415-429.

[7] A. Cavicchioli and F. Hegenbarth, 'The homotopy classification of 4-manifolds having the fundamental group of an aspherical 4-manifold', Osaka J. Math. 37 (2000), 859-871.

[8] A. Cavicchioli, F. Hegenbarth and D. Repovš, 'On the stable classification of certain 4-manifolds', Bull. Austral. Math. Soc. 52 (1995), 385-398.

[9] A. Cavicchioli, F. Hegenbarth and D. Repovs, 'Four-manifolds with surface fundamental groups', Trans. Amer. Math. Soc. 349 (1997), 4007-4019.

[10] A. Cavicchioli, F. Hegenbarth and F. Spaggiari, 'Embedding 4-manifolds with vanishing second homology', Topology Appl. 123 (2002), 313-322.

[11] M.H. Freedman, 'The topology of four-dimensional manifolds', J. Differential Geom. 17 (1982), 357-453.

[12] M.H. Freedman, 'The disk theorem for four-dimensional manifolds', in Proceed. Intern. Congress of Mathematicians, Warsaw 1983 (PWN, Warsaw, 1984), pp. 647-663.

[13] M.H. Freedman and F. Quinn, Topology of 4-manifolds (Princeton Univ. Press, Princeton, N.J., 1990).

[14] M.H. Freedman and P. Teichner, '4-Manifold topology I: Subexponential groups', Invent. Math. 122 (1995), 509-529.

[15] M. H. Freedman and P. Teichner, '4-Manifold topology II: Dwyer's filtration and surgery kernels', Invent. Math. 122 (1995), 531-557.

[16] I. Hambleton and $M$. Kreck, 'On the classification of topological 4-manifolds with finite fundamental group', Math. Ann. 280 (1988), 85-104.

[17] F. Hegenbarth, D. Repovš and F. Spaggiari, 'Connected sums of 4-manifolds', Topology Appl. 146 (2005), 209-225.

[18] J.A. Hillman, The Algebraic Characterization of Geometric 4-Manifolds, London Math. Soc. Lect. Note Ser. 198 (Cambridge Univ. Press, Cambridge, 1994).

[19] J.A. Hillman, 'Free products and 4-dimensional connected sums', Bull. London Math. Soc. 27 (1995), 387-391.

[20] J.A. Hillman, 'Complex surfaces which are fibre bundles', Topology Appl. 100 (2000), 187-191.

[21] J.A. Hillman, Four-manifolds, geometries and knots 5 (Geometry and Topology Monographs, Coventry, 2002).

[22] R. Kirby, 'Problems in low-dimensional topology', in Geometric Topology, (W. H. Kazez, Editor), Studies in Advanced Math. 2, (Georgia Intern. Topology Conf., Athens, Georgia, August 2-3, 1993) (Amer. Math. Soc., Providence, R.I., 1997). 
[23] M. Kreck, 'Surgery and duality', Ann. of Math. (2) 149 (1999), 707-754.

[24] V.S. Krushkal and R. Lee, 'Surgery on closed 4-manifolds with free fundamental group', Math. Proc. Cambridge Philos. Soc. 133 (2002), 305-310.

[25] V.S. Krushkal and F. Quinn, 'Subexponential groups in 4-manifold topology', Geom. Topol. 4 (2000), 407-430.

[26] F. Luo, 'The existence of $K\left(\pi_{1}, 1\right)$ 4-manifolds which are rational homology 4-spheres', Proc. Amer. Math. Soc. 104 (1988), 1315-1321.

[27] T. Matumoto and A. Katanaga, 'On 4-dimensional closed manifolds with free fundamental groups', Hiroshima Math. J. 25 (1995), 367-370.

[28] R. Mandelbaum, 'Four-dimensional topology: an introduction', Bull. Amer. Math. Soc. (N.S.) 2 (1980), 1-159.

[29] D.J.S. Robinson, $A$ course in the theory of groups, Graduate Text in Math. 80 (SpringerVerlag, Berlin, Heidelberg, New York, 1982).

[30] D. Ruberman, 'Invariant knots of free involutions of $\mathrm{S}^{4}$, Topology Appl. 18 (1984), 217-224.

[31] D. Ruberman, 'Seifert surfaces of knots in $\mathbb{S}^{4}$, Pacific J. Math. 145 (1990), 97-116.

[32] F. Spaggiari, 'Four-manifolds with $\pi_{1}$-free second homotopy', Manuscripta Math. 111 (2003), 303-320.

[33] F. Spaggiari, 'On the stable classification of spin four-manifolds', Osaka J. Math. 40 (2003), 835-843.

[34] E. Specker, 'Die erste cohomologie gruppe von überlagerungen und homotopie eigenschaften dreidimensionaler mannigfaltig keiten', Comment. Math. Helvetici 23 (1949), 303-333.

[35] C.T.C. Wall, Surgery on compact manifolds (Academic Press, London, New York, 1970).

[36] Z. Wang, 'Classification of closed nonorientable 4-manifolds with infinite cyclic fundamental group', Math. Res. Lett. 2 (1995), 339-344.

[37] G.W. Whitehead, Elements of homotopy theory (Springer-Verlag, Berlin, Heidelberg, New York, 1978).

Dipartimento di Matematica

Università di Modena e di Reggio Emilia

Via Campi 213/B

41100 Modena

Italy

e-mail: cavicchioli.alberto@unimo.it

spaggiari.fulvia@unimo.it 